\title{
One World; One Family; One Love
}

\author{
Geon Ho Bahn \\ Department of Psychiatry, Kyung Hee University School of Medicine, Seoul, Korea
}

To characterize this journal, the editorial committee decided to publish a special topic necessary for practitioners dealing with children's mental health in every issue, and guest editors have been invited to this end. This started with the January 2017 issue covering "Violence and Abuse." Subsequently, the April issue covered "Revised Korean practice parameter for ADHD," and the July issue covered "Impact of childhood exposure to intimate partner violence." The special topic was "Development of the Korean practice parameter for ADHD in adults" in January 2020 and "Custody evaluation in child and adolescent psychiatry in Korea" in April 2020. Going forward, we will deal with "Self-mutilation and suicide among the youth" in October 2020, "Movement disorders" in January 2021, and "Autism spectrum disorders" in April 2021.

In this issue, professor Jung-Woo Son has been invited as a guest editor to publish an article on brain imaging with pediatric psychiatry-related research materials. Bung-Nyun Kim et al. reviewed neuroimaging-based deep learning in neurodevelopmental disorders during childhood. Seungwon Chung and Jung-Woo Son presented visual perception in autism spectrum disorder from neuroimaging studies, particularly the neural underpinnings of visual detection, motion perception, and face processing. Jung-Woo Son et al. explored the differences in cognitive and empathic capabilities between adolescents and adults through an fMRI scanning study. In addition to the special topics, Donghun Oh and Keun-Ah Cheon reviewed the relations between gut microbiota and the pathophysiology, particularly social impairment and behavioral problems, of autism spectrum disorder. There are two original articles. The article by Jae Hong Park confirmed the correlation between methylphenidate treatment in children with attention-deficit/hyperactivity disorder as well as their growth through the comparison of growth markers.
The article by Tae Won Park et al. included the Korean translation of the Premonitory Urge for Tics Scale as well as the reliability and validity of the scale. It is rare for Crohn's disease patients, particularly adolescent patients, to show complications of mental illness during steroid treatment. Na Ri Kang, a child psychiatrist, and Ki Soo Kang, a pediatrician, reported a teenager with Crohn's disease who recovered from psychotic symptoms during steroid treatment.

Unlike other infectious diseases, the coronavirus disease 2019 (COVID-19) has severely impacted global health for a prolonged duration. As there have been significant restrictions on daily life, such as sports, travel, and school, psychological withdrawal and COVID-19-related stigmatization have become severe. In the meantime, the old theme of black-andwhite conflict has emerged in the U.S., leading to massive protests. The harder it is to live on the planet, the more the people have to overcome the crisis while being faithful toward their work at their respective locations.

The term of the editor-in-chief of this journal was fixed for 2 years, but was extended to 5 years for long-term project promotion and amended to allow for a second term. My 5-year term ends at the end of this year. During my first 5-year term, the editorial committee made a task force (TF), with team leader Min-Hyeon Park and team member Jae Hyun Yoo, to undertake the long-term overseas portal registration of this journal. Earlier this year, immediately before the COVID-19 outbreak, the TF sent applications to Scopus and PubMed Central (PMC), and shortly received an e-mail stating that there are no contraindications to accession to those portals at this point. While the whole world was disturbed with the COVID-19 outbreak, somewhere on the other side of the globe, somebody was doing his/her job silently. After all, the truth, "the world is one, and we are one family," as depicted in Amor Vincit Omnia ${ }^{11}$ is simple but robust.

This is an Open Access article distributed under the terms of the Creative Commons Attribution Non-Commercial License (https://creativecommons.org/licenses/by-nc/4.0) which permits unrestricted non-commercial use, distribution, and reproduction in any medium, provided the original work is properly cited.

${ }^{1}$ Amor Vincit Omnia (Love conquers all.) is a painting by the Italian artist Michelangelo Merisi da Carvaggio (1571-1610). 